\title{
DE LA PAROLE AUX VIDEOS. ORALITE, ECRITURE ET ORALITE MEDIATIQUE DANS LA PRODUCTION CULTURELLE AMAZIGH (BERBERE)
}

\section{Daniela Merolla}

University of Leiden

African Literatures

Languages and Cultures of Africa

2300 RA Leiden

The Netherlands

D.Merolla@let.leidenuniv.nl

\section{SUMMARY}

From the Spoken Word to Video: Orality, Literacy, Mediated Orality, and the Amazigh (Berber) Cultural Production

This article presents new directions in Tamazight/Berber artistic productions. The development of theatre, films and videos in Tamazight are set in the framework of the historical and literary background in the Maghreb and in the lands of Amazigh Diaspora. 
It also includes the interview with the video-maker and director Agouram Salout.

Key Words: tamazight, berber, theatre, videos, film, taqbaylit, tarifit, tachelhit, new cultural production, writing, orality

\section{INTRODUCTION}

Les modifications qui ont vu le jour dans le champ socioculturel amazigh (berbère) ont contribué à un renouvellement littéraire et artistique qui trouve expression dans les productions théâtrales et cinématographiques actuelles. Le théâtre et l'audiovisuel, en détournant les difficultés de la communication écrite entre les auteurs et le public amazigh dans un contexte encore fortement marqué par l'oralité, deviennent donc un terrain d'expérimentation artistique ainsi qu'un moyen puissant de critique sociale et de construction identitaire. Cela ne veut pas dire que la production culturelle dans l'oralité ne fonctionne pas au présent, tout au contraire les genres oraux berbères sont bien vivants. On assiste toutefois au renouveau des formes et des thèmes dans une interaction entre oralité, écriture et oralité 'médiatique' qui est réalisée par des auteurs insérés dans plusieurs circuits de production littéraire, orale et écrite. Un rôle central dans ce renouveau est évidemment joué par l'éducation scolaire et la connaissance des genres et des œuvres internationaux, ainsi que par l'exposition aux différents médias ${ }^{2}$.

\footnotetext{
${ }^{2}$ Ma recherche a lieu grâce au financement de l'Organisation Néerlandaise de la Recherche Scientifique. Une version différente et précédente de cet article a été publiée dans le Journal of North African Studies 2002. Je remercie les collègues qui ont commenté des versions préliminaires de cet article à l'Université de Leiden (NL), à l'Université de California (USA), à l'INALCO (Paris), à l'Université d'Agadir (Maroc) et à l'Université de Mainz (D).
} 


\section{TAMAZIGHT}

Il est probablement utile de rappeler à grands traits de quel champ linguistique et culturel on parle ici. La langue tamazight, ou berbère ${ }^{3}$, est une langue afro-asiatique (ou chamito-sémitique) et, différentiée en plusieurs variétés régionales, est parlée par environs $40 \%$ de la population au Maroc, par 25\% de la population en Algérie, et dans de pourcentages plus limités en Libye, au Mali, et au Niger. Reconnue au Mali et au Niger comme langue nationale, le tamazigh a obtenu très récemment quelque reconnaissance également en Algérie et au Maroc sous la pression de manifestations populaires. Cette langue reste pourtant minoritaire et politiquement dominée, avec peu d'investissements étatiques pour l'alphabétisation et pour son utilisation dans le cadre scolaire. Les différentes variétés berbères du Maghreb, tels le taqbaylit et le tachawit en Algérie ou le tarifit et le tachelhit au Maroc, ont coexisté pendant plusieurs siècles avec l'arabe largement acquis après le $\mathrm{XII}^{\mathrm{e}}$ siècle. La communication et la production culturelle orales en berbère ont ainsi coexisté avec la présence connue - et prestigieuse - de l'écriture en arabe et en particulier de l'écriture sacrée du Coran. L'utilisation des langues étant évidemment liée aux rapports sociaux, politiques et militaires entre les groupes humains, la relation hiérarchique entre une langue écrite et des langues orales n'est pas une donnée figée pour toujours ni n'est liée aux caractéristiques intrinsèques de l'une ou l'autre langue, mais elle est l'expression de processus historiques et donc changeables. La diffusion de l'arabe écrit a donc conduit dans quelques régions à une production écrite en berbère de type religieux, comme on le voit dans le cas de plusieurs

\footnotetext{
${ }^{3}$ Dès la dernière décade du vingtième siècle l'usage des termes 'Amazigh', 'Imazighen' et 'tamazight' en substitution de, ou avec, les termes 'Berbère', 'Berbères' et 'langue berbère' tend lentement à s'affirmer dans les études et en société. Ces dernières dénominations persistent pourtant dans la mesure où elles sont connues du grand public et établies depuis longtemps déjà dans les études. 'Berbère' est étymologiquement lié à un jugement péjoratif, puisqu' à travers le latin et l'arabe il est dérivé du grec barbaros qui indiquait ceux qui parlent une langue incompréhensible ou 'bégayante' pour les Grecs et qui a endossé le signifié plus commun de 'inculte' et 'non civilisé'. Le refus du regard d'autrui sur sa propre culture et la volonté de se dénommer de façon autonome a conduit plusieurs intellectuels à favoriser le choix du terme endogène 'tamazight'.
} 
manuscrits anciens en tachelhit écrits en graphie arabe ${ }^{4}$. Mais, en général, il y avait dans la plupart des régions berbérophones une production très variée de genres littéraires oraux, comme on le voit dans les collections et transcriptions du dix-neuvième siècle ${ }^{5}$, tandis que la connaissance du Coran et des œuvres édifiantes en arabe était liée à la présence des savants, des familles maraboutiques et des centres d'enseignement coranique.

Les changements sociaux et politiques, entrânés par la diffusion de l'Islam et de la langue arabe dans le Nord de l'Afrique, ont donc conduit à la formation de contextes diglossiques où des langues orales étaient utilisées pour la communication quotidienne et la production artistique (les variétés dialectales berbères ou l'arabe dialectal) ainsi que pour le commerce et la communication entre individus et groupes éloignés (l'arabe dialectal) tandis qu'une langue écrite (l'arabe classique ou coranique) était le véhicule de la religion, de la production littéraire savante et des administrations urbaines.

\section{LE COLONIALISME}

A partir de la première moitié du dix-neuvième siècle la colonisation va superposer la langue française (écrite et parlée) au contexte diglossique maghrébin et, en même temps, va stimuler l'étude des langues locales nécessaire à l'entreprise militaire ainsi qu'à l'administration des régions conquises. Cela active le 'passage à l'écrit' contemporain des langues berbères, un processus qui aura un impact symbolique élargi dans les communautés amazighophones de par la possibilité de modifier la condition de subordination historique du berbère (oral) par rapport aux langues écrites (arabe/français) ${ }^{6}$.

Cependant, il ne faut pas oublier que les études berbères se développent le long d'une double route dans le système colonial. D'un côté, les cours de linguistique berbère étaient dispensés au niveau

\footnotetext{
${ }^{4}$ L'écriture tifinagh utilisée chez les Touaregs, dérivante de l'ancien alphabet libyque, est limitée à un usage ludique ou rituel et, comme indiqué par Chaker (1984: 34), ne serve pas "de support à la mémoire collective (littéraire, institutionnelle ou historique)".

${ }^{5}$ Merolla 2000.

${ }^{6}$ Chaker 1989: 20-21.
} 
universitaire et para-universitaire, évidemment destinés à former les militaires, les administrateurs et les interprètes pour la conquête et la gestion des départements incluant des populations berbérophones. Les écoles publiques et missionnaires, de l'autre côté, n'ont jamais diffusé l'enseignement du berbère en Algérie et au Maroc. Le nom des écoles 'franco-berbères' au Maroc, par exemple, était dû au bassin de recrutement des élèves dans les régions du Moyen et Haut Atlas, mais on y enseignait exclusivement en français pour faire obstacle à la diffusion de l'arabe et de l'islam dans les pays berbérophones. La politique coloniale n'avait en effet aucun intérêt à soutenir un enseignement berbère autonome et indépendant ni la formation de berbérisants locaux. De même, après l'indépendance et jusqu'à très récemment, il n'y avait pas d'enseignement scolaire du berbère et, pendant longtemps, les études universitaires se sont développées principalement dehors du Maghreb. Cette situation était la conséquence de la politique coloniale de division entre groupes ethnolinguistiques et en même temps une expression des politiques autoritaires des gouvernements maghrébins. Dans la situation donnée, la plupart de la production écrite dans le domaine littéraire et artistique du Maghreb s'est donc développée en arabe ou en français. Les œuvres des écrivains d'origine berbère les plus connus, tels que Mouloud Feraoun, Mouloud Mammeri et Khair Eddine, ont été écrites en français et la production d'auteurs immigrés ou issus de l'immigration en Europe a été largement écrite dans les langues de ces pays ${ }^{7}$.

\section{LE PRINTEMPS BERBERE ET LA PRODUCTION LITTERAIRE ECRITE}

Le passage à l'écrit dans le domaine de la production littéraire en tamazight (langue berbère) n'a pas reçu de supports institutionnels et a donc été l'œuvre d'activités de volontariat et d'associations culturelles. Intellectuels, étudiants, chanteurs, travailleurs alphabétisés ont, au Maghreb ou dans l'émigration, utilisé et diffusé les instruments linguistiques, en particulier les systèmes de transcription, développés

\footnotetext{
${ }^{7}$ Je me réfere à la vaste production en français mais aussi à la nouvelle production en néerlandais et en italien d'auteurs d'origine marocaine.
} 
par les études universitaires berbères. A partir du 1980, on assiste à la publication des premiers romans en kabyle. C'est l'année des plusieurs manifestations contre le gouvernement en Kabylie, ce qui a été appelé le 'Printemps Berbère'. . L'entrecroisement entre création littéraire et contexte historique explique la tonalité militante des premiers romans comme Asfel (Le sacrifice rituel) et Faffa (La France) de Aliche (1981, 1986), et Askuti (Le boy-scout) de Sadi (1983), ainsi que de la collection de poèmes Isefra umehbus (Poèmes du prisonnier) de Hmed-Zayed (1981). L'engagement reste également une caractéristique des romans publiés successivement tels I $\boldsymbol{d} \boldsymbol{d}$ wass (Le jour et la nuit) de Mezdad (1990), Tafrara (Aurore) de Zénia (1995) et Si tedyant yer taye suivante les écrivains rifains révèlent une contestation engagée mais plus tournée vers l'intérieur: Mohammed Chacha aborde la rupture des tabous socio-culturels dans Rez ttabu ad d teffegh tfukt (Rompre le tabou et laisser le soleil paraître) et Mustafa Ayned dans ses récit brefs Rehriq $n$ tiri, De pijn van de schaduw, korte verhalen in het RifTamazight ${ }^{10}$ (La douleur de l'ombre, récits courts en rif-tamazight) traite avec une ironie subtile du jeu de la mémoire et des aspects absurdes de la vie quotidienne ${ }^{11}$. Le 'passage' à l'écriture exprime, d'une part, un choix d'engagement culturel et politique, et les revues associatives y ont donc joué un rôle important en donnant aux nouveaux auteurs la possibilité d'être publiés, et, d'autre part, ce passage est dû également à la scolarisation, la diffusion de plus en plus extensive des médias, et la formation d'un nouveau type de public amazighophone.

\footnotetext{
${ }^{8}$ Une telle définition est évidemment 'militante' et modelée sur le 'Printemps de Prague'.

${ }^{9}$ Par contre, les deux premiers chapitres d'un roman écrit en kabyle par une jeune femme écrivain (Merabti 1997, 1998) ne sont pas directement revendicatifs ou politiquement engagés, mais concernent les relations familiales entre hommes et femmes dans un contexte kabyle pauvre et bouleversé par les changements socio-économiques (voir le titre Yir Tagmat ou Mauvaise Fraternité).

${ }^{10}$ Le titre est bilingue berbère-néerlandais.

"Une autre tendance dans la production littéraire des années 1980 est donnée par la parution des premières collections de poèmes berbères modernes ayant l'exil comme thème central.
} 
La création contemporaine d'une littérature écrite en tamazight implique toutefois des problèmes de communication entre les auteurs 'lettrés' et leur public encore plus aigus que dans le contexte maghrébin arabophone et francophone où déjà le public qui sait lire est restreint ${ }^{12}$. Si un passage 'généralisé' à l'écrit en tamazight reste visé par les acteurs culturels, il n'y pas lieu de s'étonner qu'aujourd'hui la création artistique passe largement par des genres et des médias qui favorisent la communication orale, comme la chanson, le théâtre et les vidéos.

\section{LE THEATRE}

\section{Rite religieux}

Le théâtre qui a vu le jour récemment au Maghreb diffère des formes théâtrales déjà connues et intégrées dans les rites religieux de caractère agraire qui marquent les étapes de la vie individuelle et collective. L'usage des masques est, par exemple, répandu pendant les célébrations de la fête islamique de l'Achoura, qui célèbre la fin de l'année et qui a été absorbée dans plusieurs régions par la fête de l'Aid el Kebir célébrée dans le même période. Les descriptions ethnographiques de l'Achoura dans les régions amazighophones de la Kabylie, du Rif, du Haut Atlas et de l'Anti Atlas mentionnent la présence de deux à quinze personnages aux coutumes volumineux et portant des masqués faits de peaux d'animaux et d'écorce de calebasses. Ces masques, suivis par un cortège de musiciens, passent dans le village en chantant et font mine d'entrer de force de maison en maison pour recevoir des offrandes (des gâteaux, des amandes, des figues sèches etc.) et apporter fécondité et bien-être. Des masques typiques sont le vieil homme et l'âne, mais on retrouve aussi celle de la fillette, dans le Rif par exemple, ainsi que celle de l'esclave et du juif avec quelquefois leurs correspondants féminins dans le Haut Atlas. Les aspects théâtraux sont donnés non seulement par l'usage des masques mais

\footnotetext{
${ }^{12}$ On n'a pas encore des données sur la lecture en tamazight. Les lecteurs pour le présent, restent restreints aux circuits universitaires et de l'activisme culturel, mais on aura probablement des changements avec la récente intégration du tamazight dans les écoles au Maroc.
} 
aussi par les gestes obscènes, les chansons grivoises et l'ensemble des caractères carnavalesques du rite ${ }^{13}$.

\section{L'Halqa}

Des éléments théâtraux apparaissent également dans les séances de contage sur les marchés dites halqa, cycle ou cercle, un genre oral dans lequel le public se rassemble autour d'un conteur professionnel. La performance de l'halqa, donné essentiellement par la récitation et la gestualité du conteur, peut aussi voir la participation au jeu dramatique d'un musicien et d'un assistant, le dernier vêtu de manière bizarre et voyante, avec des éléments à la fois masculins et féminins selon Taifi (2000: 14). Si les conteurs professionnels d'aujourd'hui perpétuent une tradition ancienne, ils sont aussi insérés dans le contexte actuel caractérisé par l'interaction des genres et des médias. L'ensemble des changements socio-économiques peut engendrer le risque de disparition de cet art oral, mais la créativité de ces conteurs leur a aussi permis de jouer avec l'influence de la radio et de la télévision ${ }^{14}$. De plus c'est à ces professionnels de l'art oral que les réalisateurs des pièces et des vidéos s'adressent souvent pour travailler avec des acteurs expérimentés ${ }^{15}$.

\section{Evolution récente}

Cependant, le théâtre, dans la forme d'une pièce jouée par des acteurs dans l'espace clos d'une salle, est un genre nouveau. Si les premières pièces en arabe datent du début du $\mathrm{XX}^{\mathrm{e}}$ siècle, les expériences de spectacles de théâtre joués en berbère sont des années 1970 avec la troupe de Kateb Yacine qui, dans Mohamed prends ta valise, utilisait l'arabe dialectal et le berbère kabyle (taqbaylit) pour se rapprocher du public algérien. Dans la même période Muhand U Yehyya publiait ses adaptations en kabyle des pièces internationales avec une influence

13 'Ašura', Encyclopédie berbère, VIII, 1990: 1231-1232; Lakhsassi 1989: 31-39; Laoust 1921; Musso 1970; Servier 1985.

${ }^{14}$ Voir le discours inaugural de Juan Goytisolo à la proclamation de l'insertion de la place de Djamaa el-Fna à Marrakesh (où acrobates et conteurs donnent leurs spectacles) dans la liste du patrimoine mondial de l'UNESCO le 15 mai 2001.

${ }^{15}$ Voir l'entretien avec Agouram Salout ci-dessous. 
majeure du théâtre européen (Beckett, Brecht, Molière, Pirandello). Dans une interview accordée en 1985, U Yehyya expliquait que traduire et recréer des pièces était pour lui une étape du développement de la tradition littéraire en kabyle ainsi que le moyen de renouveler la production artistique sans "mimer stupidement les autres" mais en tant que "reconstruction [de l'ouvrage] au moyen de matériaux qu'il [l'adaptateur] puise dans son environnement culturel". Le ton typique de ces adaptations est celui de la dérision qui vise le totalitarisme étatique ainsi que, et peut-être encore plus, les stéréotypes, les idées enracinées, les faiblesses et les illusions du public kabyle. Dans le même entretien, U Yehya disait ensuite que publier sur cassette audio et vidéo était ce qui correspondait "le mieux aux exigences de l'heure" à cause de l'analphabétisme ${ }^{16}$. Egalement, le choix d'utiliser dans ses pièces la langue de tous les jours, avec les emprunts à l'arabe et au français, était lié pour lui à la communication avec son public.

\section{LA DERNIERE DECENNIE}

\section{Taqbaylit}

Au cours de la dernière décennie, la production théâtrale kabyle en Algérie et en France a augmenté et un grand nombre de pièces ont été jouées par des acteurs professionnels et amateurs ${ }^{17}$. Malgré les longues années de guerre interne et les deux dernières années de révolte populaire en Kabylie, plusieurs troupes amateurs et estudiantines persistent courageusement dans leur activité. Evidemment, on constate en même temps une hémorragie des forces vers les pays de l'émigration qui offrent plus de sécurité sinon plus de possibilités de travail.

\footnotetext{
${ }^{16}$ U Yehya affirmait également qu'il avait élaboré les textes à l'écrit et que les questions liées à la production par l'écrit restaient dans ses préoccupations (p.57). Certaines adaptations de U Yehya ont été publiées comme textes écrits, voir U Yehya 1974, 1976, 1986, 1987.

${ }^{17}$ En tout manque de données, on dira qu'il y a plusieurs dizaines des pièces qui ont été jouées en tamazight ou en forme bi-trilingue.
} 
De manière générale, on dira que si le théâtre amateur kabyle reste très lié à la langue maternelle, à la fois pour le type d'auditoire et l'engagement en faveur du berbère, les compagnies professionnelles utilisent l'arabe ainsi que le français et le kabyle ${ }^{18}$. C'est le cas par exemple du célèbre acteur Mohamed Fellag qui, en Algérie, a créé ou joué des pièces dans ces trois langues ${ }^{19}$. Aujourd'hui en exil, et donc porté à monter ses spectacles en français, Fellag ponctue ses 'oneman-shows' de mots et d'expressions tirés de dans ses deux autres langues, ce qui est un des éléments de son grand succès auprès du public 'monolingue' français ainsi que chez les immigrés plurilingues. On retrouve aussi cette stratégie linguistique chez Moussa Lebkiri, acteur et écrivain issu de l'immigration kabyle à Paris. L'auto-dérision, l'humour et l'ironie socioculturels, qui jouent souvent avec le multilinguisme des auteurs et du public, caractérisent ce théâtre multivocal et multilangue. Les motifs et les thèmes des pièces de Fellag sont sociaux et politiques à partir de Babor Australia à Djurdjurassic bled (un jeu de mots entre le titre du film Jurassic Park et le bled - le village - du Djurdjura, le nom des montagnes de la Kabylie centrale), et en passant par Sinni, une adaptation en kabyle des Emigrés de Slawomir Mrozek.

Ces spectacles, recréés en français après 1995, racontent avec un humour corrosif l'histoire et le présent tragique de l'Algérie ainsi que la vie de l'émigration, les difficultés quotidiennes, les tabous, les histoires de folie ordinaire, le désarroi individuel et collectif ${ }^{20}$.

\footnotetext{
${ }^{18}$ Sur le multilinguisme dans le théâtre kabyle, voir Siagh 1991.

${ }^{19}$ Mohamed Fellag, né en 1950 à Azzefoun en Kabylie, a habité à Alger dès l'âge de huit ans. Après ses études à l'Ecole nationale des arts dramatiques d'Alger, il a été engagé dans plusieurs spectacles et est devenu ensuite le directeur du théâtre de TiziOuzou. Dés 1978 il participe à plusieurs expériences théâtrales en France, au Canada et aux Etats-Unis, avant de retourner en Algérie où il travaille avec un succès extraordinaire dans le théâtre mais aussi pour le cinéma. En 1995 il est contraint de se réfugier en France à cause de la guerre civile et des assassinats de plusieurs intellectuels. On peut trouver des informations biographiques et artistiques sur Fellag sur le site de Theatre-on-line (www.theatreonline.com). Il a publié son premier roman Rue des petites daurades en 2001 et le recueil de nouvelles C'est à Alger en 2002 (chez Jean-Claude Lattès).

${ }^{20}$ Babor Australia, actualisée dans Un bateau pour l'Australie en 2002, s'est inspiré par exemple d'un épisode absurde dans l'Algérie des années 1990: des gens se présentent devant l'ambassade d'Australie après la diffusion de la rumeur qu'un bateau
} 
Les pièces burlesques de Moussa Lebkiri ${ }^{21}$ proposent des thèmes plus intimes mais également brûlants et parlent de la mémoire de l'enfance kabyle et immigrée dans Une Etoile dans l'ceil de mon frère et Kif-kif piment ou touchent aux idées de moralité et d'amoralité dans Jardin des roses et des soupirs, fondé sur des contes érotiques arabes du XIII ${ }^{\mathrm{e}}$ et $\mathrm{XV}^{\mathrm{e}}$ siècle $^{22}$.

\section{Tachelhit}

Au Maroc, les pièces théâtrales de Moumen Al Safi, publiées dans les années 1980, sont considérées comme le début du théâtre amazigh moderne. Safi nous a dit qu'il avait commencé à écrire en tamazight pour donner un signe de l'existence de sa langue maternelle après avoir assisté à un cours universitaire dans lequel le berbère avait été étiqueté 'langue morte' à l'instar du latin ${ }^{23}$. Ecrits dans le tachelhit de la région de Casablanca, ses textes métaphoriques et poétiques mettent en jeu les questions existentielles ainsi que l'oralité et l'écriture en berbère. Ussan semmiḍnin ou Les jours froids présente un village forcé à réfléchir sur lui même et sa propre façon de vivre quand un matin le soleil ne se lève pas, tandis que la question de la langue est traitée dans Tighrit tabrat (Lecture d'une lettre) à travers la recherche

\footnotetext{
australien allait emmener les chômeurs algériens et leur donner un emploi et un logement (et un kangourou, comme le dit Fellag).

${ }^{21}$ Moussa Lebkiri, né en 1952 à Beni Chabane en Kabylie, a grandi dans la périphérie parisienne. Il commence à travailler comme aide-photographe, passe ensuite aux marionnettes pour se consacrer enfin au théâtre en 1976 avec la compagnie Nedjma, dont il est le fondateur, en se produisant dans la rue pendant le Festival d'Avignon. Informations sur l'activité artistique de Moussa Lebkiri à l'adresse http: //nedjma7.free.fr/Menu_moussaLebkiri/moussa_cv.html\#Jardin (avec une belle galerie de ses marionnettes). Il a publié plusieurs recueils de ses contes modernes et, en 2002, le roman Bouz'louf, Tête de mouton (chez l'Harmattan).

${ }^{22}$ Voir aussi la présentation de son dernier travail, Au bout du conte...elle danse, avec Saliha Bachiri (danse) et Aïni Iften (conte et chant): "Hadjila est vedette contrariée par son mektoub, possédée par son chitane artistique elle est Wanted, recherchée dans tout le continent berbère...".

${ }^{23}$ Moumen Al Safi est né en 1948. Ses parents étaient de Ait Temzal et il a étudié initialement à l'école coranique du village. Il a poursuivi ses études à Rabat et est actuellement avocat à Casablanca.
} 
déçue d'une vieille femme ${ }^{24}$. Ensuite, plusieurs acteurs et compagnies d'amateurs sont devenus actifs dans la dernière décennie du $X X X^{e}$ siècle dans la région de Casablanca. On peut mentionner par exemple les acteurs comiques Abdellatif Atif et Ahmed Nassih, qui travaillent avec la compagnie 'Tifawine' bien connue du public amazighophone, le musicien et acteur Brahim Hannoudi et sa femme Fatima Joutam, qui ont joué plusieurs pièces de théâtre avec le groupe 'Timitar', et enfin Rachid Boulmazghi (Aslal), monologuiste qui connaît bien les représentations carnavalesques par tradition familiale.

\section{Tarifit}

La naissance du théâtre d'auteur en tarifit, le berbère parlé dans la région du Rif dans le nord du Maroc, est ensuite attribué à Azreoual Fouad qui a écrit sept pièces de théâtre et un roman dans les années $1990^{25}$. Ses textes ne sont pas publiés, mais toutes ses pièces ont été mises en scène grâce à la collaboration des associations culturelles locales. Son premier monodrame met en jeu la recherche identitaire d'un intellectuel amazigh qui passe en revue l'histoire des langues et cultures du Maghreb, du tamazight à l'arabe, au latin et au grec. Dans d'autres pièces, traitant plus directement des difficultés de la vie au pays et dans l'émigration, la critique sociale touche aux aspects absurdes de la quotidienneté avec des influences de Kafka et Gogol, comme dans Aryur-inu Iizzen (Mon âne bien aimé) où un âne obtient un diplôme et fait carrière dans l'administration. Dans un entretien ${ }^{26}$, Azeroual Fouad indique un itinéraire artistique semblable à celui de U Yehyya pour le théâtre kabyle. Son intérêt pour le théâtre, connu grâce à la production et l'adaptation en arabe, l'a en fait porté à traduire des

\footnotetext{
${ }^{24}$ La vieille femme reçoit une lettre de son fils émigré en France, le premier contact avec lui depuis vingt-cinq ans. Après de vaines recherches, elle trouve finalement un garçon qui peut la lire, mais ni lui ni elle peuvent ne comprendre un mot parce que la lettre est écrite en arabe.

${ }^{25}$ Azroual Fouad est né à Nador dans une famille originaire de Ait-Touzine (entre AlHoceima et Tamssamane). Il a fait ses études dans sa ville natale et ensuite à l'université de Oujda. Il est professeur d'arabe au collège de Nador et membre de l'Institut Royal de la culture amazighe crée en 2001. Il prépare une thèse sur la réception dans le spectacle populaire au Rif.

${ }^{26}$ Nous tenions à remercier Azroual Fouad que nous avons rencontré à Nador en octobre 2000 .
} 
textes en tarifit avant de se décider à écrire et monter des pièces nouvelles. De même, le choix du théâtre n'est pas seulement lié à son propre intérêt artistique, mais aussi à la nécessité de communiquer avec le public ${ }^{27}$. La réaction du public amazighophone a été très positive ${ }^{28}$.

"J'écris des pièces qui sont très faciles et comiques et qui parlent des problèmes sociaux qui sont proches de notre public. Je parle de souffrances et de la pensée commune pour que le public puisse accepter cette nouvelle forme d'art... Ce qui importe, c'est de faire entrer un nouveau genre dans notre culture amazigh pour publier dans notre langue et dire nos pensées".

"Le public ici nous fait du bien, il est vraiment pour le tamazight, mais comme je vous ai dit le support matériel et moral nous manque".

Il s'agit évidemment encore d'un théâtre amateur et, selon Fouad, il sera donc nécessaire d'améliorer l'aspect esthétique des textes et de la mise en scène ainsi que la professionnalité des acteurs qui sont issus, pour la plupart, de troupes de théâtre scolaires. Ces groupes d'écoliers et d'étudiants, très motivés et souvent guidés par des metteurs en scène semi-professionnels, auront effectivement besoin de pouvoir se perfectionner ailleurs ${ }^{29}$.

Si les pièces de Safi marquent le début du théâtre d'auteur tamazight en tant que textes écrits et publiés, des acteurs semi-professionnels avaient déjà commencé auparavant à jouer en tamazight des pièces déjà connues en arabe ou des adaptations de sketches et de contes. C'est le cas de Farouk Aznabet qui, ayant joué pendant plusieurs années dans le théâtre amateur arabe, avait porté en scène des pièces

${ }^{27}$ Fouad: "Il y a aussi un autre problème. Je vous donne un exemple: deux frères viennent ici en entendant dire qu'il y aura une rencontre sur le tamazight. Ils sont très intéressés, mais ils veulent quelque chose qui soit direct, pas de contes, pas de livres parce qu'ils ne lisent pas.... Plus direct, alors c'est pour cela qu'ils viennent voir le theâtre".

${ }^{28}$ Azroual Faouad et Farouk Aznabet nous ont dit qu'une fois le public applaudissait et battait les pieds en rythme si fortement après la représentation 'Allal en Allemagne' (l'histoire d'un émigrant en Europe) qu'ils ont presque craint que la salle - qui était située dans une maison de jeunesse ancienne - ne s'effondre.

${ }^{29}$ C'est le cas du groupe Zegangan (Nador) organisé par Ahmed Allaoui et Bilal Azufoud. 
en tarifit pour mieux réussir à entrer en contact avec le public amazighophone. Toutefois, les conditions générales à la fin des années 1970 n'étaient pas favorables à la poursuite de cette expérience et, encore sans contacts avec les mouvements associatifs berbères, il a continué à jouer en arabe jusqu'aux années quatre-vingt-dix quand il a décidé de monter des pièces 'solo' en tarifit ${ }^{30}$. L'arme critique de la dérision et l'ironie caractérisent la plupart des pièces de Aznabet, qu'elles soient situées dans le présent ou dans un espace-temps épique et fabuleux, par exemple dans La cité des rêves et dans Nunja ${ }^{31}$. Pour créer ses pièces, Farouk Aznabet a collaboré avec Azroual Fouad, qui réside à Nador, et avec un autre écrivain, Ahmed Ziani, qui travaille dans le Rif et en Europe. Aznabet lui même a joué ses pièces non seulement dans le Rif, mais aussi dans plusieurs villes marocaines, belges et néerlandaises. Comme pour les écrivains et les acteurs kabyles, on constate une continuité de la production culturelle entre le pays d'origine et l'émigration rifaine qui s'est orientée vers la Belgique, les Pays-Bas et, plus récemment, l'Espagne.

\section{QUESTIONS OUVERTES}

Une question débattue par les auteurs et les acteurs concerne la langue des nouvelles productions en tamazight, c'est à dire, soit l'utilisation de la langue quotidienne avec les emprunts à l'arabe et au français, soit la purification lexicale de la langue en récupérant les mots anciens et désuets et en rénovant le vocabulaire dialectal avec des néologismes fondés sur les racines et les mots présents dans les autres variétés régionales. D'un côté, pour adhérer au réalisme narratif, on veut faire parler les personnages dans la langue qui correspond le mieux à leur position socio-culturelle; de l'autre côté, on veut contrer le risque de perte de vitalité du tamazigh confronté à la concurrence d'autres langues, dominantes puisqu'elles sont langues nationales, par un effort

\footnotetext{
${ }^{30}$ Entretien personnel.

${ }^{31}$ Dans le premier travail, deux anciens rois berbères (Jugurtha et Taqfarinas) s'en prennent l'un à l'autre et s'adressent à Numidia, la terre imaginaire des Imazighen. Dans Nunja, la fille de l'ogresse est représentée seulement par ses tresses et sa voix, mais on ne la voit jamais: c'est la vie des femmes qui restent toujours chez elles et la télévision est leur seule forme de communication avec le monde extérieur.
} 
volontariste de recherche linguistique et culturelle. De plus, il faut se rappeler que la question de la langue du roman et du théâtre a une histoire particulière dans le contexte maghrébin et africain puisqu'elle s'est lourdement colorée de références nationalistes et identitaires pendant (et après) la période de la colonisation européenne.

La participation restreinte des actrices au théâtre amazigh, en particulier des jeunes femmes, est ensuite déplorée par plusieurs écrivains et compagnies marocaines ${ }^{32}$. Bien qu'il nous semble que la situation évolue, certainement dans les pays de l'émigration, le cœur du problème réside dans la coutume sociale qui exige des femmes un maintien modeste et déconseille leur présence devant un public mixte. Des membres de la compagnie Tifawine de la région du Souss nous ont dit qu'ils ont tenu plusieurs réunions pour convaincre les jeunes actrices et leurs familles d'entreprendre ou de continuer l'activité théâtrale. De même, la jeune actrice rifaine Laïla Elazrak mentionne dans un entretien les discussions familiales sur son choix de faire du théâtre $^{33}$. Dans la pièce Nunja, mise en scène par Farouk Aznabet plusieurs fois au Maroc et aux Pays-Bas, le fait que la fille montre seulement ses très longues tresses a aussi bien servi comme escamotage pour éviter les difficultés de trouver une jeune actrice, et une fois, y il a quelques années, le rôle féminine dans la pièce La cité des rêves a été joué par une étudiante hollandais $\mathrm{e}^{34}$.

Tous les acteurs et les écrivains interviewés sont enfin d'accord sur le fait que le développement du thêâtre amazigh est lié aux moyens institutionnels et matériels, qui manquent à cause de la politique d'exclusion linguistique encore mise en vigueur. Laïla Elazrak explique par exemple que son expérience majeure s'est effectuée grâce au théâtre de langue française, où l'utilisation de langues locales est très sporadique, et que l'engagement personnel pour poursuivre des études et au niveau financier est actuellement exorbitant pour ceux qui voudraient se

\footnotetext{
${ }^{32}$ L'écriture féminine en berbère au Maroc est encore plus restreinte. Par exemple, nous avons rencontré seulement trois femmes auteurs, Aicha, Fatima Bouziane et Fatima Bouchane, et une seule a publié (en arabe). Ces écrivains ont créé des récits modernes fondés sur des thèmes de contes berbères. Fatima Bouchane, la plus jeune, a essayé la traduction en berbère d'un roman et l'écriture d'un scénario.

${ }^{33}$ Voir Rif Bulletin 2000: 21-22.

${ }^{34}$ Entretien personnel.
} 
consacrer sérieusement au théâtre amazigh ${ }^{35}$. Le théâtre professionnel au Maroc reste donc le domaine des productions en arabe et en français qui sont étudiées dans les instituts artistiques et qui sont subventionnées par l'Etat.

\section{Le théâtre émigré}

Dans l'émigration, la situation peut évoluer vers la professionnalisation grâce aux possibilités de spécialisation et de supports financiers. Le trajet du régisseur et acteur Chaib Massaoudi est ainsi emblématique: après d'autres expériences de travail aux PaysBas, il a décidé de se consacrer au théâtre et a bien réussi ${ }^{36}$. Diplômé comme régisseur à l'institut universitaire pour les Arts de Utrecht (Hoge School voor de Kunsten), il a fondé en 1998 le groupe théâtral Amazigh Stichting (Fondation Amazigh), et plus récemment le groupe Appolius $^{37}$. Au Maroc, il a participé à la réalisation de La masque de Alexandre Puskin (Pouchkine) par l'Ecole des beaux-arts de Rabat avec l'utilisation de chansons en tamazight ${ }^{38}$. Au cours des deux dernières années il a mis en scène deux pièces tragi-comiques, Taslit et Roméo (en néerlandais) et Rabiaa, Buzeyyan et le permis de séjour (en tarifit avec sous-titres en néerlandais), qui ont obtenu des critiques positives et un accueil chaleureux aux Pays-Bas. Grâce à une subvention de la fondation néerlandaise Prins Claus, ce dernier travail a aussi été joué à Ceuta, l'enclave espagnole au Nord du Maroc, et après un grand succès auprès du public, la troupe a obtenu l'autorisation d'effectuer une tournée dans le Nord du Maroc ${ }^{39}$. Les pièces ont été écrites par Omar Boumazzough qui parvient à réunir des thèmes internationaux et du patrimoine berbère dans la pièce Taslit et Roméo, jouée de manière convaincante entre autres par l'écrivain et musicien Mustapha Ayned, et qui touche à la critique politique dans

\footnotetext{
${ }^{35}$ Voir l'entretien avec Laïla Lazrak.

${ }^{36}$ Voir Rif Bulletin 2000: 10-13.

${ }^{37}$ Avec Oufa Ouatah, Luisa Boussattach, Taeib El Maach, Said El Marssi et Nassim El Mahi.

${ }^{38}$ En collaboration avec Nocman Awragh, régiste d'origine rifaine qui travaille en Espagne. Voir l'entretien avec Moussaoudi.

${ }^{39}$ Voir Schaap 20-9-2002 et 23-9-2002 (également sur le 'site' de Wijbrand Schaap: home01.wxs.nl/ wmschaap/artikelen/diversen).
} 
Rabiaa, Buzeyyan et le permis de séjour en traitant par lironie et l'absurde la situation des émigrés rifains et de leur langue ${ }^{40}$. Maintenant, Chaib Massaoudi est en train de mettre en scène des pièces de théâtre en coopération avec Abdelkader Benali, un écrivain qui à reçu plusieurs prix littéraires aux Pays-Bas et dont le roman Bruiloft aan zee (Noce à la mer) a été traduit en sept langues ${ }^{41}$.

Avant même d'être représentée, Rabiaa, Buzeyyan et le permis de séjour était bien connue dans le Rif puisqu'un enregistrement du texte sur cassette, envoyé pour obtenir le permis de jouer la pièce au Maroc, avait trouvé sa voie dans le circuit informel et était devenu très populaire dans les cafés de la région ${ }^{42}$. Le succès de cette pièce est typique de l'interaction entre théatre et oralité médiatique dans une situation politique fermée envers la production en tamazight. Ce n'est pas en effet un cas isolé, mais une caractéristique forte de la production culturelle amazigh 'd'auteur'. Les écrivains et les acteurs mentionnés non seulement diffusent volontiers leurs pièces grâce aux enregistrements sur cassettes, mais presque tous travaillent également dans la production de vidéos. Par exemple, une des premières pièces jouée par la troupe Tifawine dans les années 1980, Tagodi, est devenue fort populaire après sa production en vidéo en 1995 . Que les vidéos soient 'officielles' ou des copies illégales, elles soutiennent grâce à leur distribution la communication entre auteurs, acteurs et public plus efficacement que le théâtre ou que les publications écrites qui demeurent l'apanage d'intellectuels et d'activistes.

\footnotetext{
${ }^{40}$ Deux histoires tragiques, mais enrichies de traits hilarants : Taslit et Roméo combine et actualise, dans le contexte de la migration, le thème shakespearien de l'amour impossible entre les enfants des deux familles rivales en Italie, Capuleti et Montecchi, et celui de l'amour également contrasté entre Asli et Taslit, enfants de deux villages rivaux, et qui, dans la légende berbère, se transforment en deux sources dont les eaux se réunissent pour former une seule rivière; Rabiaa, Buzeyyan et le permis de séjour traite des mésaventures d'un rifain qui donne sa mère à l'imam du village et émigre pour se marier avec Rabiaa et obtenir le convoité permis de séjour en Europe, mais qui retourne chez lui dans un cercueil.

${ }^{41}$ Noces à la mer (trad. C.Auchard, éd. Albin Michel, 1999) a obtenu le prix français du Meilleur Roman Étranger en 1999. Le dernier roman de Benali, De langverwachte (L'enfant tant attendu, 2002) a obtenu le prix néerlandais Libris Literatuur en 2003.

${ }^{42}$ Interview, voir Schaap 2002.
} 


\section{Vidéos et Films}

L'entretien avec le cinéaste et chanteur Agouram Salout, dont nous reproduisons une partie ci-dessous, décrit clairement les horizons d'attente ainsi que la pratique de la production audiovisuelle en tachelhit au Maroc. Avec son film Bu Tfunast (L'homme à la vache), sorti en 1993, Salout a été un des premiers réalisateurs de vidéos en berbère tachelhit (Maroc). Le thème est tiré d'une histoire bien connue, celle d'un brigand qui veut vendre sa vache et devenir un honnête homme, et ce film vidéo a obtenu un succès inattendu. La production audiovisuelle semble répondre à une demande diffusée entre plusieurs couches de la population amazighophone: elle a été en fait accueilli avec un grand enthousiasme dans le monde des mouvements associatifs culturels comme par le public urbain et rural amazighophone. Malgré les grandes difficultés de financement, diffusion et réalisation professionnelle, entre 1993 et 2001, Salout a mis en scène dix-huit films seul ou en coopération, tandis qu'au moins une trentaine des vidéos en tachelhit ont été produites par d'autres réalisateurs. Entre autres, un long métrage a été achevé récemment par une jeune femme, Fatima Boubekdi, qui vient du milieu du théâtre et du cinéma arabe. Aujourd'hui on peut trouver des copies de films vidéo en tachelhit sur tous les marchés marocains comme dans plusieurs villes de France, de Belgique et des Pays-Bas ${ }^{43}$. Des titres connus sont par exemple Tagodi (Le chagrin, 1995) de Ahmed Badoui avec la troupe Tifawine, Tassaste (Le problème, 1994) de Agouram Salout avec Brahim Hanoudi et Fatima Joutan, Moker (1996) de Ahmed Larbi et Agouram Salout avec Fatima Taboukhouchte, Tamghart worgh (La femme en or, 1992) de Lhoussaine Bounizakam avec la troupe Tifawine, et Tihiya (Biographie de F. Tabamrante, 1994) de Ahmed Larbi et Mohamed Walkader avec la chanteuse Fatima Tabamrante. La plupart des histoires dans ces films sont situées dans une ambiance rurale et mettent en jeu des relations familiales et d'amour donnant dans la farce plutôt que dans le drame, ce que l'on pourrait presque qualifier de 'soap' rural, et se différencient

\footnotetext{
${ }^{43}$ Comme dans le cas du théâtre, on n'a pas des données. Toutefois, on dénombre au moins une cinquantaine de titres de vidéos offerts dans les magasins à Casablanca (et environs) et dans les marchés d'Amsterdam. Une courte cinématographie berbère est dans Fodil 1997.
} 
ainsi nettement de la production des premiers films du cinéma en berbère kabyle (Algérie).

La production en berbère kabyle, plus limitée, concerne des films pour le grand écran (cinéma) dont la réalisation est le résultat de la coopération de plusieurs partenaires, de différentes sources de financement et de longues années de persévérance de la part des réalisateurs et acteurs ${ }^{44}$. Le premier film, La colline oubliée, Tawriit itwattun (1994) de Abderrahmane Bouguermouh, est l'adaptation d'un des romans les plus célèbres - et controversés - de l'écrivain Mouloud Mammeri. Problèmes existentiels, d'amour et d'identité, dans un monde en changement sont vécus dans un village kabyle de l'avant guerre de libération ${ }^{45}$. La vie et l'honneur dans le monde villageois colonial sont repris dans Machacho, Il était une fois de Belkacem Hadjadj et dans La montagne de Baya, Adrar $n$ Baya (1997) de Azzedine Meddour ${ }^{46}$. Tous ces films comportent une tonalité lyrique et dramatique et le dernier en particulier a un caractère légendaire. Bien que situés dans un espace-temps révolu, celui de la colonisation et d'un monde traditionnel presque a-historique, ces films communiquent un message actuel sur la nécessité du respect des individus et de leurs aspirations ainsi qu'une dimension épique du monde berbère et l'amour pour la langue et la culture amazigh.

Aujourd'hui, la différence entre les films de cinéma en kabyle et les vidéos en tachelhit pourrait être simplifiée en termes d'intellectuel vs. populaire. Les films de cinéma sont des productions coûteuses fondées sur des textes littéraires, avec des aspirations esthétiques et culturelles liées à la revendication berbère. Les vidéos sont des productions au budget limité et, bien qu'elles aient certainement des ambitions artistiques, sont plus directement orientées vers le marché local et tendent donc à reproduire le discours social de la communauté de destination, en particulier de la classe urbaine moyenne de Casablanca ou Rabat et sa perception de la vie rurale. Une caractéristique que les

\footnotetext{
${ }^{44}$ Par exemple, Azzedine Meddour avait commencé à s'activer pour le film Adrar $n$ Baya presque dix ans avant sa réalisation.

${ }^{45}$ Pour la critique littéraire au roman voir Mortimer 1982 et Bonn 1985. Sur l'œuvre littéraire et scientifique de Mammeri voir aussi ltinéraires et contacts de cultures 1992 et Awal 1998.

${ }^{46}$ Le double titre indique que ces films ont été présentés avec des sous-titres en français.
} 
films de cinéma et les vidéos ont toutefois en commun est l'interaction entre écriture, théâtre et musique dans leur réalisation. Dans tous les cas, les acteurs des films viennent du monde du théâtre et de la musique, plusieurs musiciens, chanteurs et chanteuses étant recrutés par les régisseurs ou ayant eux-mêmes réalisé des productions audiovisuelles.

Les difficultés dans la production et la diffusion, comme le manque de données et de recherche statistique sur l'alphabétisation en tamazight et la production artistique, sont indications de la position minoritaire du tamazight. Toutefois, les pièces de théâtre, les films et les vidéos, qu'il soient explicitement engagés ou non dans les revendications linguistiques et culturelles berbères, diffusent l'idée qu'il est possible de créer en tamazight et de se libérer de la subordination aux langues écrites de la communication internationale.

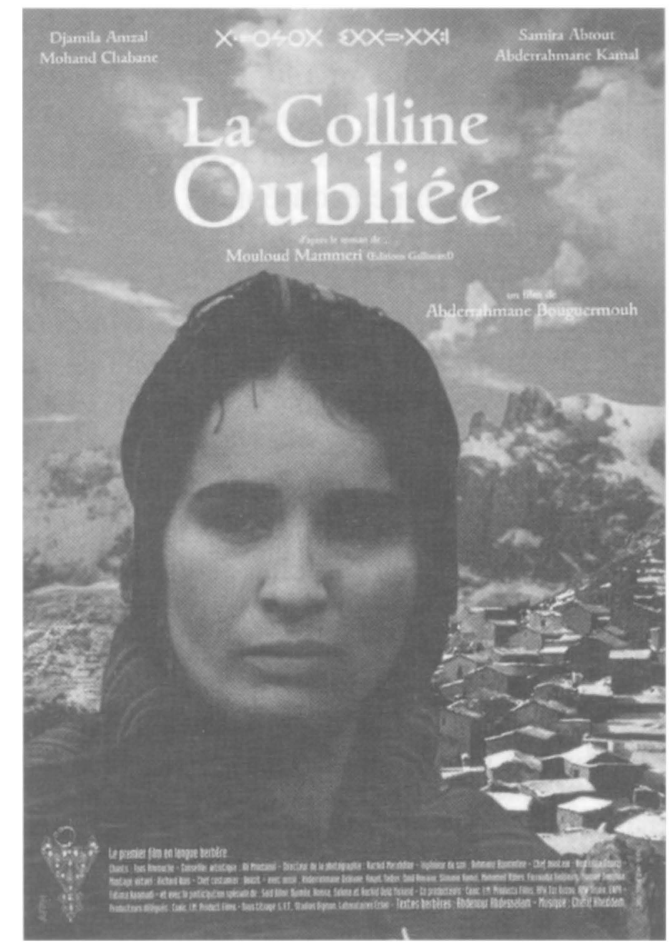




\section{Interview. Agouram Salout et les films vidéos en tachelhit $^{47}$.}

Agouram Salout : "Cela ne fait pas longtemps que j'ai commencé à m'intéresser au cinema. Mon premier film est de 1992 [...] Après l'école, je faisais le disc-jockey dans une boîte de nuit. En 1973 il y avait des acteurs connus et des réalisateurs qui venaient chez nous, ici à Casablanca, et j'ai travaillé avec eux quand il y avait un film. De 1973 à $1980 \mathrm{j}$ 'avais un groupe musical, Archach, et on a commencé à travailler avec des réalisateurs des vidéos. Quelques fois j'ai fait l'acteur [...] Je suis allé en France, pour une interview à la radio, et il y avait beaucoup de gens qui demandaient pourquoi il n'y avait pas de cinéma berbère. Quand je suis rentré de France en 1992 j'ai pensé faire un film en berbère. J'ai pris une histoire connue, $\mathrm{Bu}$ tfunast et les quarante voleurs, un peu comme Ali Baba et les quarante voleurs, mais ce n'est pas la même histoire. J'ai commencé à solliciter la Warda vision qui a décidé de produire ce film et on a trouvé qu'il y avait beaucoup d'intérêt dans le marché. Alors, quand les gens ont vu ce film, plusieurs ont commencé à travailler avec moi, comme Touriah ou Ali Hamama, Omar Siez, Mimouch, des grands acteurs au Maroc qui faisaient les films en arabe et maintenons travaillent avec moi. Jusqu'à maintenant j'ai fait dix-huit films. Je travaille aussi avec d'autres, on ne peut pas être le seul qui fait le tournage. Ce n'est pas bien."

Est-ce que c'est difficile de faire des films en berbère? Est-ce que vous avez assez d'acteurs?

S.: Il y a beaucoup d'acteurs à l'halqa de Jamalfa, de Marrakech ${ }^{48}$. On commence avec Jamalfa parce qu'il n'y a pas encore d'acteurs professionnels [...] C'est très difficile de faire du cinéma parce que les acteurs ne savent pas exactement ce qu'on fait. Tu prends par exemple un acteur de Jamalfa. Je lui dis comment il doit faire, mais en concret c'est quoi le cinéma? Ils savent faire du théâtre, mais ils ne savent pas comment faire du film. Par exemple, je lui dis: "allez" une première fois, une deuxième fois, une troisième, une quatrième et lui me dit "pourquoi ça ?".

Est-ce que vous écrivez les scénarios vous-même ou demandez-vous de l'aide à des écrivains?

S.: Non, non vraiment. J'utilise les histoires, les sujets des écrivains, puis j'écris les scénarios. J'ai tourné seulement trois scénarios qui ne sont pas à moi.

\footnotetext{
47 Agouram Salout, chanteur du groupe musical Archach, est aussi connu comme 'Agouram Archach'. L'entretien a eu lieu à Casablanca, le 28 septembre 2001, avec la participation de la journaliste cinématographique Cristiana Paternó.

${ }^{48}$ Par exemple, l'acteur de 'halqa' Da Hmad est devenu célèbre pour son interprétation dans le film vidéo $B u$ Tfunast de Salout.
} 
J'ai utilisé l'histoire de Tabar Amran, une histoire de Ibrahim Oufdi et une autre de Ibrahim ou Chmouch, par exemple, mais ce sont de vieilles histoires.

\section{Est-ce que vous le tournez en trente-cinq millimètres?}

S.: Non. La pellicule pour le film cinéma coûte très cher, on n'a pas encore les moyens. Pour les films en arabe il y a le centre cinématographique, et ils ont donné des subventions, mais pour les films en berbère non, pour nous les réalisateurs et les acteurs non. Maintenant il y a des pièces de théâtre en berbère, et si l'on projette le film au cinéma il est sûr et certain que la salle sera pleine. Chaque fois qu'on a fait l'avant première pour les journalistes, la salle était pleine; et quand on a fait le premier festival du film berbère c'était plein, tous les jours de la semaine. Au festival, j'ai obtenu le premier prix avec Tafunast. Il y a le marché, il y a le public, mais pas de financement... [Pour la vente] le problème est qu'il y a plusieurs pirates [...]. Il y a avait sept maisons de production en berbère, mais maintenant il en reste seulement deux.

\section{Est-ce que ce sont des contes?}

S.: Il ne s'agit pas vraiment de contes. Par exemple, Tassast : le scénario est de Brahim Hannoudi. Il est un acteur, mais il a écrit aussi trois pièces théâtrales et les scénarios de deux films. Boukdi Brahim aussi, il a déjà travaillé avec Badaoui dans des pièces thêâtrales [...] Il y a des écrivains berbères qui ont publié des pièces théâtrales, mais ce sont des choses très difficiles pour en faire des scénarios [...] On a fait des films jusqu'à maintenant typiquement berbères, au bled, avec les questions de mariages, de cœur, parce que les berbères qui habitent à Rabat, dans les villes ont besoin de voir, ils aiment le folklore. Alors on a commencé comme ça. Par exemple, le film Moker, c'est l'histoire de deux frères jumeaux, un qui est méchant et l'autre qui est gentil. Le frère méchant habite dans le désert, l'autre habite au village. On l'a tourné avec 63 acteurs et en trois épisodes, trois cassettes vidéo. Je crois qu'on pourra en faire un film en arabe pour la T.V. marocaine en dix-huit épisodes.

Est-ce que vous avez vu La colline oubliée? Est-ce qu'il y a des trais en commun entre vos productions et ce type de film kabyle?

S.: Oui, je l'ai vu. Il y a une grande différence, parce que je ne peux pas commencer avec un grand film. Il faut commencer par quelque chose qui fait rigoler. C'est pour cette raison que j'ai commencé par Bu tfunast, L'homme à la vache. Une moitié du film est tournée au bled, c'est pour distraire les gens, pour les faire rire [...]. Dans mes films il y a toujours du dramatique et du comique, il faut les deux; on ne peut pas pleurer tout le temps, il faut aussi rire, alors je fais toujours de la comédie. 
Pour ce qui concerne la langue tamazight, quelle langue utilisez-vous?

S.: Le tamazight de Agadir. C'est toujours la langue normale. Par exemple on dit 'merci', ou je dit 'azul' ['hallo', mot utilisé dans les associations] et un autre dit 'eh?' et l'on répète 'salam aleikum', ou je dis 'tanmirt' ['merci', 'adieu', mot utilisé dans les associations] et un autre dit quoi? et je lui dis 'tanmirt', comme sukran, pour faire comprendre les mots à tout public berbère. Quand j'étais avec le groupe Archach, pour les chansons, on nous demandait souvent "mais qu'est-ce-que vous disiez...?". Par exemple, même des Imazighen ne savaient pas ce que veux dire 'timuzra', c'est-à-dire la liberté, un mot qui vient du terme amazigh, les hommes libres. Alors je parle toujours dans un berbère qui est compréhensible.

Je voudrais remercier les écrivains, les acteurs et les réalisateurs qui ont bien voulu me rencontrer. Mes remerciements sincères pour l'accueil et le soutien vont ensuite à plusieurs collègues et amis, et en particulier à Abdelkader Aissati, Mohamed Amrhar, Fatima Al Ayoubi, Mohamed Al Ayoubi, Zohra et Farouk Aznabet, Mohamed Amir, Anir, Hassan Belkassm, Brahim Baouch, Latifa Douch, Mena Lafkioui, Abderrahmane Lakhsassi, Abdallah El Mountassir, Fatima Al Mourabiti, et l'association Tamaynut.

\section{BIBLIOGRAPHIE}

'AŠURA', Encyclopédie berbère, VIII, 1990: 1231-1232.

AWAL, La dimension maghrébine dans l'oeuvre de Mouloud MAmmeri, vol. 18, 1998.

BONN, C., Le roman algérien de langue française, L'Harmattan, Paris, 1985.

CHAKER, S., Textes en linguistique berbère, Editions du CNRS, Paris, 1984.

CHAKER, S., Berbères aujourd'hui, l'Harmattan, Paris, 1989.

FODIL, R., Cinématographie berbère, Tiziri, nov. 1997: 42-43.

Itinéraires et Contacts de cultures, Hommage à Mouloud Mammeri, vol. 15/16, 1992

LAKHSASSI, A., Réflexions sur la mascarade de Achoura, Signes $d u$ présent (Rabat), 6, 1989: 31-39.

LAOUST, E., Noms et cérémonies des feux de joie chez les Berbères du Haut et de l'Anti-Atlas, Hespéris, I, 1921: 3-66. 
LAZRAK, L., (Entretien avec Lailla Lazrak), Theater: interview met Laïla Lazrak, actrice uit Rif, Rif Bulletin, Association Amazigh Syphax, Utrecht, Pays-Bas, année 2, no. 2, 2000: 21-22.

MASSAOUDI, C., (Entretien avec Chaïb Massaoudi), Amazigh Theater in Nederland, gesprek met Chaïb Massaoudi, Rif Bulletin, association Amazigh Syphax, Utrecht, Pays-Bas, année 2, no. 1, 2000: 10-13.

MERABTI, F., Yir Tagmat, Tizir, nov. 1997: 36-40; et no. 10, jan. 1998: 35-38 (manuscrit complet inédit).

MEROLLA, D., Littérature berbère: textes en prose, dans U. Baumgardt et A. Bounfour (Ed.), Panorama des littératures africaines, L'Harmattan/Inalco, Paris, 2000: $15-26$.

MEROLLA, D., Digital Imagination and the 'Landscapes of Group Identities': the Flourishing of Theatre, Video and 'Amazigh Net' in the Maghrib and Berber Diaspora, The Journal of North American Studies, vol. 7, no. 4, 2002: 122-131.

MORTIMER, M., Mouloud Mammeri, écrivain algérien, Sherbrooke, Naaman, 1982 Musso, J.-Cl., Masques de l'Achoura en Grande Kabylie, Libyca, XVIII, 1970: 269274.

SAFI, M. Al-, Ussan semmidnin, (Les jours froids), Ed. Al-Andalus, Casablanca, 1983.

SCHAAP, W., Berbers toneelsucces sluitstuk van Marokkaans festival, Utrechtsnieuwsblad, 20-9-2002.

SCHAAP, W., Stadsschouwburg pakt uit met El Fraaja, Utrechtsnieuwsblad, 23-92002.

SERVIER, J., Tradition et civilisation berbères, Rocher, Monaco, 1985: 409-416, 447-461.

SIAGH, Z., Le théâtre amateur en Algérie: choix et usage des langues, International Journal of the Sociology of Language, 87, 1991, pp. 71-82.

U YEHYY, M., Llem-ik, ddu d uq̣ar-ik (adapt. de l'Exception et la règle de B. Brecht), Tala, Paris, 1974.

U YEHYA, M., Aneggaru ad yerr tabburt ('Le dernier ferme la porte', adapt. de La décision de B.Brecht), Univ. Paris VIII, GEB, Paris, 1976.

U YEHYYA, M., (Entretien avec Muḥend U Yehyya), Entretien à la revue Tafsut, Tafsut (Tizi-Ouzzou), no. 10, 1985: 43-64. 
U YEHYA, M., Muhend u Ca'aban (adapt. de Le Ressussité de Lu Hsun), Tiddukla, no. 6-7, $1986: 16-17$; et no. $8,1987: 10-12$.

U YEHYA, M., Si Leḥlu (adapt. de Le médecin malgré lui de Molière), Awal, no.2, 1986: 145-156; et no. 3, 1987: 147-190. 


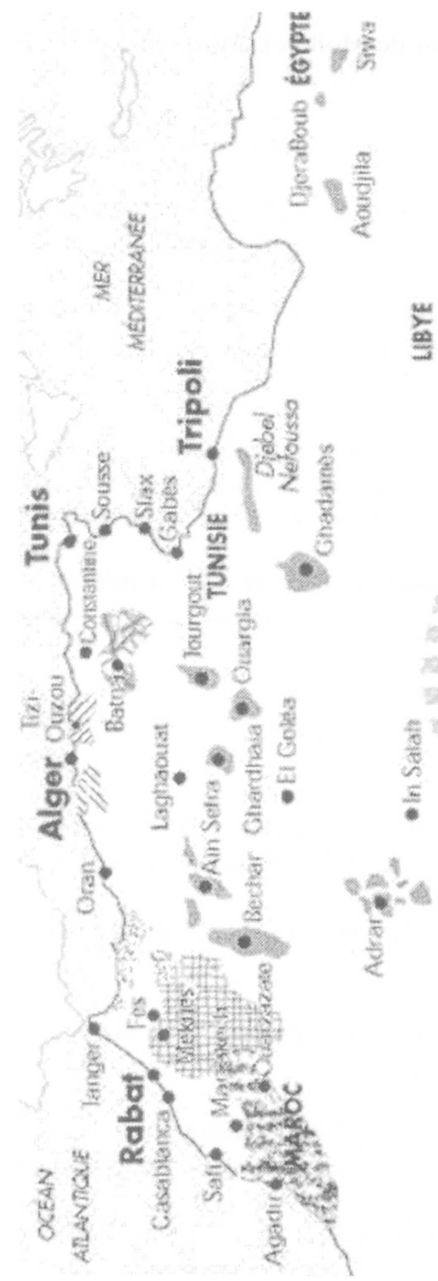

鉴
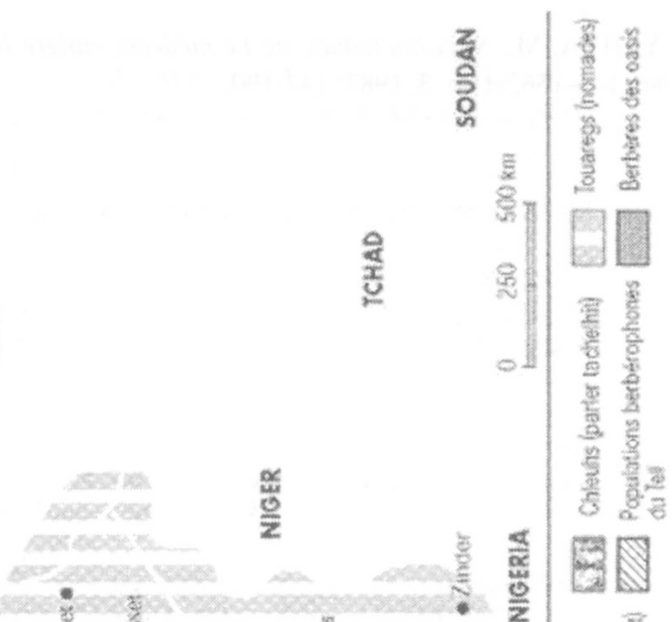

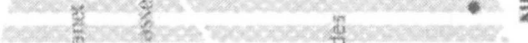

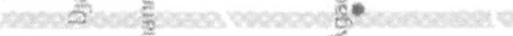

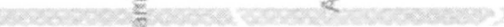

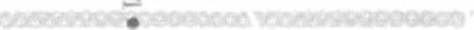

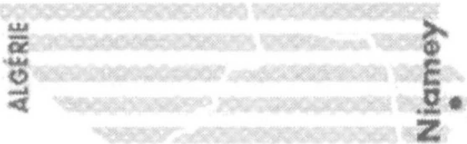

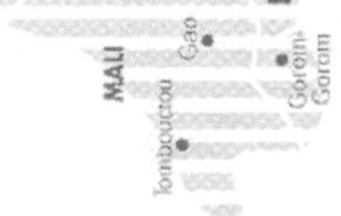
竞, 욜

$\frac{\frac{m}{2}}{\frac{\hat{\alpha}}{\mathrm{c}}}$ 